\title{
Gestão de pessoas em propriedades leiteiras: uma análise no Agreste de Pernambuco
}

\author{
Maria Rosicléa Teles Honorato* \\ Suellen Arlany Silva Gomes \\ Daniela Moreira de Carvalho****
}

\begin{abstract}
Resumo
O objetivo do artigo consiste em avaliar as práticas de gestão de pessoas dentre as técnicas de treinamento, remuneração e avaliação de desempenho na região Agreste do estado de Pernambuco. Implementar estratégias de gestão de pessoas é fundamental para o sucesso das organizações, visto que as mudanças necessárias na empresa só terão efetividade e legitimidade a partir do envolvimento das pessoas. Para tanto, foram entrevistados 41 funcionários e 30 gestores de cidades localizadas na bacia leiteira do estado de Pernambuco. Os resultados apontaram inexistência das técnicas da gestão de pessoas na região de estudo, baixo índice de treinamentos, insatisfação dos funcionários e alto índice de informalidade. A ausência de feedback entre gestores e funcionários resultou em $85 \%$ de insatisfação dos funcionários. Contudo, é importante ressaltar que ter foco com a gestão de pessoas, estimular o comprometimento da mão de obra com as propriedades e gerar motivação e capacitação são fatores primordiais para o crescimento da pecuária leiteira.
\end{abstract}

Palavras-chave: Mão de obra. Gestão. Recursos humanos. Pecuária de leite.

* Graduanda em Zootecnia, Universidade Federal do Agreste de Pernambuco. E-mail: teles.rosicleia@ gmail.com

** Mestranda em Engenharia de Produção, Universidade Federal de Pernambuco. E-mail: suellenarlany@gmail.com

**** Professora Doutora em Agronegócios, Universidade Federal do Agreste de Pernambuco. E-mail: dmcoop@gmail.com 


\section{Introdução}

Inserida no agronegócio, a cadeia agroindustrial do leite é reconhecida como uma das mais importantes do setor sob as óticas social e econômica. Presente em todo o território nacional, estima-se que 558 microrregiões geográficas brasileiras produzam leite. Além disso, a atividade atua na formação de emprego de aproximadamente 4 milhões de pessoas que trabalham diretamente na atividade (CLEOCY, 2019).

Em 2018, a produção brasileira de leite chegou a 33,8 bilhões de litros, e 13\% do leite produzido nacionalmente são oriundos da Região Nordeste (CNAE; IBGE, 2019). A cadeia do leite é a principal atividade para dezenas de municípios do estado de Pernambuco. No Agreste do estado, encontra-se a mais importante bacia leiteira, responsável por $80 \%$ da produção de leite do estado (SANTOS, 2019). Nesse aspecto, podemos observar a grandeza e a importância da pecuária de leite para o estado de Pernambuco, onde o investimento no capital humano ainda é escasso. Ter foco com a gestão de pessoas, estimular o comprometimento da mão de obra com as propriedades e gerar motivação e capacitação são fatores primordiais para o crescimento da pecuária leiteira.

No presente, existe uma literatura substancial sobre o uso das práticas de gestão de pessoas e seu impacto no desempenho de organizações em outros setores. Porém, pouca informação estruturada é encontrada na literatura sobre gestão de pessoas em propriedades leiteiras. Isso resulta em uma compreensão um pouco limitada de como as práticas podem ter impacto no desempenho das propriedades, sobretudo considerando a diversidade de gestão observada entre diversas regiões (ULLAH; ZHENG, 2014).

Nesse sentido, a pesquisa teve como objetivo avaliar as práticas de gestão de pessoas em propriedades leiteiras da região Agreste do estado de Pernambuco, pois identificar elementos sobre o perfil dos funcionários e proprietários, a remuneração, os treinamentos e desafios na obtenção e manutenção da mão de obra, bem como as demandas dos funcionários, torna-se importante, devido ao fato de tais elementos serem fundamentais para o avanço da gestão de empresas rurais, uma vez que padrões de gestão eficazes impactam de maneira decisiva na sua sustentação e na competitividade do desenvolvimento econômico da organização e do setor. 


\section{Referencial teórico}

Ocupando a $3^{\text {o }}$ posição do ranking mundial de produção de leite, o Brasil abriga um dos maiores rebanhos produtivos do mundo, com aproximadamente 37 milhões de cabeças, estimando um efetivo de 16,4 milhões de vacas ordenhadas em 2018. Do total de animais ordenhados, o Sudeste segue com o maior efetivo ordenhado nacional, representando $29,2 \%$, enquanto o Sul representa $20,6 \%$ e o Nordeste, $20,4 \%$, ocupando o segundo e o terceiro lugar, uma diferença percentual pequena no rebanho, embora com diferenças significativas em termos de produtividade (CNAE; IBGE, 2019). No Nordeste, o estado da Bahia atualmente lidera o ranking, produzindo 870.281 mil litros/ano, enquanto Pernambuco ocupa a segunda posição, produzindo 795.698 mil litros/ano (CNAE; IBGE, 2019).

O Agreste de Pernambuco é composto principalmente por pequenas e médias propriedades, a maioria destas com características de agricultura familiar. Elas são, em sua maioria, gerenciadas de forma empírica, com pouco ou sem nenhum conhecimento de gestão. O baixo nível tecnológico aplicado nas propriedades e a falta de gestão mais profissionalizada conferem ao segmento produtivo indicadores técnicos aquém das suas reais potencialidades (MONTEIRO et al., 2007).

São vários os fatores que limitam a gestão de pessoas nas empresas rurais. Um exemplo é a caracterização da produção agropecuária de tempos atrás ter sido realizada por serviços braçais, conduzida por mão de obra escrava, que aos poucos foi sendo substituída por imigrantes italianos, os quais utilizavam implementos manuais de tração animal para realização das atividades. Devido a essa estrutura escravagista, nos dias de hoje, tem-se um histórico de mão de obra barata e pouco qualificada que ainda persiste em alguns locais. Outro fator que limita as empresas rurais para implementar a gestão de pessoas na empresa rural são as exigências legais previstas na Consolidação das Leis Trabalhistas (CLT) (BRASIL, 1943), pois o empregador tem dificuldades de se adequar às regulamentações exigidas para o setor. Além disso, o processo de administração que acomete as propriedades é tipicamente autoritário-paternalista, não permitindo a melhoria do profissional, levando as empresas rurais a uma alta taxa de rotatividade (YAMAUCHI, 2017). Implementar estratégias de gestão de pessoas é fundamental para o sucesso das organizações, visto que as mudanças necessárias na empresa só terão efetividade e legitimidade a partir do envolvimento das pessoas (DA SILVA et al., 2016). 
Diante do contexto exposto, os aspectos básicos da gestão de pessoas nas empresas rurais são treinamento, remuneração e avaliação de desempenho. Tais aspectos, visualizados como parceiros da empresa, fornecem conhecimentos, habilidades e, sobretudo, inteligência especializada, auxiliando a empresa no alcance do sucesso.

As modificações que estão ocorrendo no cenário da economia têm causado grande impacto no agronegócio brasileiro. A busca por produtos diversos causa uma fragmentação de mercado, crescendo a exigência dos consumidores por qualidade.

O funcionário é um aliado extremamente importante, pois o resultado da qualidade da produção está mediante sua responsabilidade. A Instrução Normativa 77 (IN-77) do Ministério da Agricultura, Pecuária e Abastecimento (Mapa), de novembro de 2018, exige uma melhora significativa na qualidade do leite produzido pelo produtor, pois estabelece parâmetros de procedimentos e ações para boas práticas agropecuárias, com a finalidade de obter leite de qualidade e seguro ao consumidor. As normas exigidas pela IN-77 englobam a organização da propriedade, de instalações e equipamentos, bem como formação e capacitação dos responsáveis pelas tarefas cotidianas. $\mathrm{O}$ artigo $6^{\circ}$ da $\mathrm{IN}-77$ estabelece que os produtores tenham como parte de seu programa de autocontrole um plano de qualificação dos fornecedores do leite, o qual deve contemplar assistência técnica e gerencial, bem como a capacitação dos funcionários (BRASIL, 2018; DA SILVA et al., 2016).

É sabido que o sistema de ordenha adotado nas propriedades leiteiras é de grande importância para a tomada de decisões no quesito qualidade, seja a ordenha manual ou mecânica, a forma de manipulação dela influenciará diretamente a qualidade do leite. Para uma ordenha adequada, existe a necessidade de uma mão de obra treinada, entretanto, talvez esse seja um dos principais problemas enfrentados pelos produtores de leite, sabendo que é a principal etapa do processo de produção que demanda a capacitação da mão de obra (PEIXOTO, 2016).

$\mathrm{O}$ treinamento prepara o funcionário com o objetivo de promover a aquisição de habilidades, regras, atitudes e comportamentos, aperfeiçoando o desempenho da pessoa ocupante do cargo. Isso resulta numa melhor adequação entre as características dos funcionários e as exigências da função. É importante entender a necessidade de seguir todas as etapas de um processo do treinamento, pois apenas dessa forma os resultados esperados poderão ser atingidos. $\mathrm{O}$ primeiro aspecto a ser tratado dentro da organização é o amadorismo da administração, visto que 
técnicas de treinamento ainda são pouco exploradas no setor agropecuário (RUSSI; COSTA E SILVA; ZÚCCARI, 2009).

Associados à gestão estratégica de pessoas, os sistemas de remuneração e avaliação de desempenho são cruciais para estimular os funcionários na obtenção do aumento da produtividade e para cumprir os objetivos e metas da organização. Não há dúvidas de que é essencial incluir os sistemas de remuneração e avaliações de desempenho entre as prioridades de políticas de gerenciamento das empresas rurais, com o objetivo de estabelecer equilíbrio interno na empresa, sem essa política, a motivação e o desempenho no ambiente de trabalho tornam-se baixos. $\mathrm{Na}$ remuneração, o equilíbrio externo também deve ser avaliado, correlacionando a remuneração para os cargos com as médias do mercado, dentro de uma região geográfica. Para os cargos operacionais, são considerados fatores como formação escolar, experiência, responsabilidade por contatos e por resultados, riscos de acidentes, condições ambientais e esforço físico resultante da execução das atividades de rotina. Já o processo de avaliação de desempenho é facilitado pela utilização de elementos que permitam uma análise qualitativa e quantitativa, possibilitando pontuar fatores como qualidade, rendimento, zelo, comportamento, assiduidade, entre outros (GONÇALVES; STEFANO; BACCARO, 2017).

\section{Procedimentos metodológicos}

Com o intuito de avaliar a gestão de pessoas nas empresas rurais, foi realizada uma pesquisa de caráter exploratório a partir de uma abordagem qualitativa por meio de questionários, buscando-se analisar o perfil das propriedades leiteiras e dos funcionários com base nas técnicas de gestão de pessoas. Nesta pesquisa, foram entrevistados 41 funcionários e 30 gestores de propriedades leiteiras de médio porte, distribuídos em 10 cidades da bacia leiteira do Agreste de Pernambuco: Águas Belas, Alagoinha, Bom Conselho, Buíque, Garanhuns, Itaíba, Pedra, Saloá, São Bento do Uma e Venturosa.

A análise das entrevistas é descrita considerando a percepção de gestores e funcionários. Foram realizadas entrevistas com 30 gestores, cujo maior percentual da pesquisa se concentrou nas cidades de Bom Conselho, Pedra e Venturosa, importantes produtoras de leite em 2018. Bom Conselho teve uma produção de $46.731 \mathrm{mil} /$ litros/leite; Pedra, uma produção de 49.876 mil/litros/leite; e Venturosa, $35.485 \mathrm{mil} /$ litros/leite. Além de se destacarem na produção de leite, os municípios 
abrigam importantes laticínios (ASSOCIAÇÃO BRASILEIRA DAS INDÚSTRIAS DE ALIMENTAÇÃO, 2018).

Os dados constituem-se como primários, obtidos através da pesquisa de campo, em que foram colhidas informações ao longo dos meses de agosto a novembro de 2019. A amostra utilizada caracteriza-se como não probabilística por conveniência, pois leva em consideração as pessoas que aceitaram participar da pesquisa (funcionários e gestores), assim, foi possível comparar, examinar e conferir diferentes dados e informações relevantes, buscando identificar posicionamentos divergentes e convergentes em relação à visão teórica abordada.

\section{Resultados e discussão}

\section{Entrevista aos gestores}

Os sistemas de produção da pecuária brasileira variam entre regiões, a Tabela 1 mostra o sistema de criação predominante nas propriedades da região de estudo, onde foi possível observar que 76\% das propriedades analisadas são compostas do sistema de produção semi-intensivo. Para Abrantes et al. (2016), o sistema de produção semi-intensivo é composto por instalações mais complexas, em que há necessidade de salas de ordenhas e sistemas de conservação para estocagem do leite, instalações separadas para novilhas/vacas e bezerros, currais de alimentação e de espera, entretanto, o sistema semi-intensivo utilizado na região de estudo se dá pela falta de alimento a pasto.

Tabela 1 - Sistemas de produção na região de estudo

\begin{tabular}{l|c|c}
\multicolumn{1}{c|}{ Sistema de produção } & N & Percentual (\%) \\
\hline Extensivo & 3 & 10,00 \\
Semi-intensivo & 23 & 76,67 \\
Intensivo & 4 & 13,33 \\
\hline Total & 30 & 100 \\
\hline
\end{tabular}

Fonte: dados da pesquisa, 2019. 
De Camargo (2018) relata, em sua pesquisa, que produtores do estado de São Paulo alegam ter dificuldade na obtenção de mão de obra que queira trabalhar na pecuária de leite, devido à intensa jornada de trabalho. Os dados desta pesquisa nos mostram o contrário, em que $100 \%$ dos proprietários entrevistados na região de estudo disseram não ter dificuldades para obtenção da mão de obra. Em relação à quantidade de pessoas que trabalham nas propriedades, $33 \%$ dos entrevistados disseram ter em média de 3 a 6 funcionários, como mostra a Tabela 2. O número de funcionários por propriedade varia de acordo com a quantidade de animais, entretanto, eles são distribuídos em atividades específicas ou em rotação de atividades.

Tabela 2 - Número de funcionários por propriedade

\begin{tabular}{l|c|c}
\hline \multicolumn{1}{c|}{ Número de funcionários } & N & Percentual (\%) \\
\hline 0 a 3 & 8 & 26,67 \\
3 a 6 & 10 & 33,33 \\
6 a 10 & 7 & 23,33 \\
Mais de 10 & 5 & 16,67 \\
\hline Total & 30 & 100 \\
\hline
\end{tabular}

Fonte: dados da pesquisa, 2019.

Em relação à gestão de pessoas, foi perguntado aos gestores sobre as suas percepções em relação à satisfação dos funcionários em trabalhar em suas propriedades. Como mostra a Tabela 3, 80\% relataram que seus funcionários estão satisfeitos, pois eles "não reclamam", existindo boa relação entre "patrão e funcionário". Ainda, foi relatado que eles são considerados parceiros da atividade e que a rotatividade de funcionários na propriedade é quase inexistente, diferente da percepção dos funcionários que será discutida posteriormente. Quando questionados sobre folgas e férias dos colaboradores, $76 \%$ dos entrevistados informaram que seus funcionários possuem uma folga plena na semana, relação 5:1 (a cada cinco dias de trabalho uma folga). Já quanto às férias, apenas $36 \%$ dos proprietários fornecem férias aos colaboradores, sendo estas de 30 ou 15 dias. Em relação às reuniões, $53 \%$ relataram fazer reuniões quinzenais ou mensais. As reuniões, na maioria das vezes, são feitas apenas quando é identificado algum problema na propriedade ou no dia de pagamento. 
Tabela 3 - Percepção dos gestores em relação a satisfação dos funcionários, benefícios como folga e férias e práticas de reuniões com funcionários na propriedade

\begin{tabular}{l|c|c|c|c|c|c|c|c} 
& \multicolumn{2}{c|}{ Satisfação } & \multicolumn{2}{c|}{ Folga } & \multicolumn{2}{c|}{ Férias } & \multicolumn{2}{c}{ Reuniões } \\
\cline { 2 - 9 } & $\mathrm{n}$ & $\%$ & $\mathrm{n}$ & $\%$ & $\mathrm{n}$ & $\%$ & $\mathrm{n}$ & $\%$ \\
\hline Sim & 24 & 80,0 & 23 & 76,6 & 11 & 36,7 & 16 & 53,3 \\
Não & 6 & 20,0 & 7 & 23,4 & 19 & 63,3 & 14 & 46,7 \\
\hline Total & 30 & 100 & 30 & 100 & 30 & 100 & 30 & 100 \\
\hline
\end{tabular}

Fonte: dados da pesquisa, 2019.

Contudo, os gestores citaram a importância de realizar reuniões, relatando que por meio delas é possível ter a troca de ideias e experiências, tornando a propriedade um espaço para estudos e divulgação de conhecimentos, o que implica no crescimento de toda a equipe, melhorando a qualidade do serviço prestado pelos funcionários.

Em relação ao quesito treinamento, foi possível observar que apenas $30 \%$ dos proprietários investem na capacitação de seus funcionários (Tabela 4).

Tabela 4 - Percentual de propriedades que possuem assistência técnica e fornecem treinamentos aos funcionários

\begin{tabular}{l|c|c|c|c}
\cline { 2 - 4 } & \multicolumn{2}{c|}{ Treinamento } & \multicolumn{2}{c}{ Assistência técnica } \\
\cline { 2 - 5 } & $\mathrm{n}$ & $\%$ & $\mathrm{n}$ & $\%$ \\
\hline Sim & 9 & 30,0 & 17 & 56,67 \\
Não & 21 & 70,0 & 13 & 43,33 \\
\hline Total & 30 & 100 & 30 & 100 \\
\hline
\end{tabular}

Fonte: dados da pesquisa, 2019.

O baixo índice de treinamento, segundo os entrevistados, ocorre devido ao desinteresse dos funcionários e à indisponibilidade de cursos em horários que sejam compatíveis com a jornada de trabalho das propriedades. A partir dos resultados desta pesquisa, vale ressaltar que, recentemente, o Mapa, através da IN-77 (BRASIL, 2018), estabeleceu boas práticas agropecuárias que devem ser implementadas nas propriedades, com isso, o plano de qualificação de fornecedores de leite deve contemplar no mínimo a capacitação dos funcionários, seguida de assistência técnica na propriedade, para que se possa assegurar a qualidade do leite que está sendo produzido.

No que diz respeito à avaliação de desempenho dos funcionários, como mostra a Figura 1, foi possível observar que 39\% dos produtores não possuem procedimentos 
para avaliar tal prática e $26 \%$ avaliam apenas o rendimento dos funcionários de maneira informal. Conforme Yamauchi (2017), produtores geralmente avaliam o desempenho de seus funcionários de maneira verbal e sem formalidades, por isso, é necessária a utilização de um registro de ocorrências, que contenha campos para anotações tanto negativas quanto positivas, para a tomada de decisões que tornem a avaliação mais transparente e com credibilidade.

\section{Perspectiva dos funcionários}

O perfil dos funcionários é predominantemente de participação masculina, com $95 \%$ dos entrevistados, distribuídos entre as atividades de ordenhador, tratorista e trabalhador rural. O gênero feminino corresponde a apenas $5 \%$ do total, distribuídos nas atividades de secretaria e bezerreiro. Ambos os gêneros possuem faixa etária entre 19 e 50 anos (Tabela 5).

Tabela 5 - Distribuição da faixa etária dos funcionários entrevistados

\begin{tabular}{l|c|c}
\hline \multicolumn{1}{c|}{ Faixa etária } & $\mathrm{n}$ & $\%$ \\
\hline Até 18 & 0 & - \\
19 a 30 & 19 & 46 \\
31 a 50 & 22 & 54 \\
Acima de 50 & 0 & - \\
\hline Total & 41 & 100 \\
\hline
\end{tabular}

Fonte: dados da pesquisa, 2019.

Quanto ao nível de escolaridade dos funcionários, verificou-se que $34 \%$ dos entrevistados informaram ter o ensino fundamental completo e $17 \%$ ter o segundo grau completo; não foi verificado analfabetismo em nenhum entrevistado. Vale salientar que pessoas com menor grau de escolaridade tem maior dificuldade de aceitar novas tecnologias e assimilar informações relacionadas a práticas de manejo, etc., o grau de escolaridade é importante, pois permite que o funcionário acompanhe os avanços dos sistemas. Assim, o primeiro item a ser verificado é se os funcionários sabem ler e escrever de maneira satisfatória.

A Tabela 6 permite verificar a percepção dos funcionários em relação a sua satisfação em trabalhar na propriedade, folgas, férias e reuniões. Do quesito satisfação, 
85\% dos entrevistados não se sentem satisfeitos com seu trabalho. Quando questionados sobre o porquê da insatisfação, eles relataram o fato de sua remuneração ser baixa em relação à jornada de trabalho intensa e a falta de estrutura na propriedade, que possibilite melhores condições de trabalho, além da insatisfação com o gerenciamento das propriedades, referente ao autoritarismo dos gerentes/proprietários, pois não têm a oportunidade de opinar para a melhoria das suas funções em relação a estruturas e tecnologias que possibilitem uma melhor forma de trabalhar.

Tabela 6 - Percepção dos funcionários em relação a satisfação, benefícios como folga e férias e práticas de reuniões com funcionários na propriedade

\begin{tabular}{l|c|c|c|c|c|c|c|c} 
& \multicolumn{2}{c|}{ Satisfação } & \multicolumn{2}{c|}{ Folga } & \multicolumn{2}{c|}{ Férias } & \multicolumn{2}{c}{ Reuniões } \\
\cline { 2 - 9 } & $\mathrm{n}$ & $\%$ & $\mathrm{n}$ & $\%$ & $\mathrm{n}$ & $\%$ & $\mathrm{n}$ & $\%$ \\
\hline Sim & 6 & 15 & 34 & 83 & 24 & 59 & 26 & 63 \\
Não & 35 & 85 & 7 & 17 & 17 & 41 & 15 & 37 \\
\hline Total & 41 & 100 & 41 & 100 & 41 & 100 & 41 & 100 \\
\hline
\end{tabular}

Fonte: Dados da pesquisa (2019).

A percepção dos funcionários se mostra oposta à percepção dos gestores, conforme descrito na Tabela 3, em que $80 \%$ dos gestores consideram que seus funcionários estão satisfeitos. Essa percepção oposta sinaliza a falta de um canal de comunicação com o funcionário, que permita ao gestor perceber sinais de insatisfação.

Além disso, a Tabela 6 nos mostra que 83\% dos funcionários relataram ter folgas plenas na semana na relação 5:1 (a cada cinco dias de trabalho uma folga), dado que corrobora os dados apresentados na Tabela 3, do mesmo quesito questionado ao gestor, estando de acordo com artigo $1^{\circ}$ da Lei $n^{\circ}$ 605/1949 (BRASIL, 1949).

Quando questionado o quesito reuniões, 63\% dos entrevistados disseram ter reuniões pelo menos uma vez ao mês. Mas, não são reuniões de rotina, só acontecem quando necessário, devido à ocorrência de algum problema na propriedade, fato também mencionando pelo gestor, como demonstra a Tabela 3.

Para Pereira, Do Vale e Mancio (2004), pequenos problemas passavam despercebidos e causavam prejuízos, além de não ser possível identificar a insatisfação dos funcionários, essa situação foi parcialmente superada por 79\% das propriedades de bovinos de corte no Triângulo Mineiro, onde os gestores procuraram realizar reuniões com os funcionários ao longo do ano, para debater problemas internos. 
Ao analisar se os funcionários já participaram de algum tipo de treinamento, $54 \%$ dos entrevistados disseram que sim, como mostra a Tabela 7. A diferença de percentual deve-se por uma fazenda ter mais funcionários que as outras. Eles participaram de pelo menos um treinamento durante seu tempo de trabalho na propriedade. Os treinamentos mencionados foram técnicas de descorna, manejo de ordenha e inseminação artificial, que não são treinamentos rotineiros, e isso faz com que os funcionários possuam poucas habilidades em determinada função.

Tabela 7 - Distribuição de funcionários que já participaram de um tipo de treinamento na propriedade

\begin{tabular}{l|c|c}
\hline \multicolumn{1}{c|}{ Treinamento } & $\mathrm{n}$ & $\%$ \\
\hline $\mathrm{Sim}$ & 22 & 54 \\
Não & 19 & 46 \\
\hline Total & 41 & 100 \\
\hline
\end{tabular}

Fonte: dados da pesquisa, 2019.

Para Malanski, Hostiou e Ingrand (2016), funcionários sem qualificações ou com poucas habilidades técnicas são comumente atribuídos para tarefas rotineiras, não possuindo habilidades para resolução de problemas. Através do treinamento, pode-se perceber as habilidades individuais e as aptidões para orientar com mais assertividade, o importante é nunca deixar o funcionário à margem das informações, e sim considerá-los como um fator produtivo que precisa ser reciclado para melhor execução de suas tarefas.

Quanto à remuneração, esta varia de acordo com a função dos colaboradores, como demonstra a Tabela 8. Foi possível observar que, a depender do cargo, a remuneração média varia de $\mathrm{R} \$ 490,00$ a $\mathrm{R} \$ 1.600,00$.

Tabela 8 - Variação da remuneração dos funcionários por cargo

\begin{tabular}{l|c}
\hline \multicolumn{1}{c|}{ Cargos } & Remuneração \\
\hline Trabalhador rural & $\mathrm{R} \$ 800$ a $\mathrm{R} \$ 1.600$ \\
Tratorista & $\mathrm{R} \$ 950$ a $\mathrm{R} \$ 980$ \\
Ordenhador & $\mathrm{R} \$ 918$ a $\mathrm{R} \$ 1.600$ \\
Secretária & $\mathrm{R} \$ 490$ \\
Técnico & $\mathrm{R} \$ 1.300$ \\
Outros & $\mathrm{R} \$ 980$ \\
\hline
\end{tabular}

Fonte: dados da pesquisa, 2019. 
A informalidade é um dos desafios enfrentados pelo trabalhador rural. Segundo estudo publicado em 2014 pelo Departamento Intersindical de Estatística e Estudos Socioeconômicos (DIEESE), a informalidade no campo está entre as mais altas do mercado de trabalho. Só no estado de Pernambuco, a informalidade chegou a $62 \%$ em 2014 (Tabela 9).

Tabela 9 - Porcentagem de funcionários que trabalham de carteira e recebem por produção

\begin{tabular}{l|c|c}
\cline { 2 - 3 } & Trabalham de carteira assinada & Recebem por produção \\
\cline { 2 - 3 } & $(\%)$ & $(\%)$ \\
\hline Sim & 39 & 10 \\
Não & 61 & 90 \\
\hline Total & 100 & 100 \\
\hline
\end{tabular}

Fonte: dados da pesquisa, 2019.

Os dados obtidos corroboram os dados apresentados pelo (DIEESE). Esta pesquisa mostrou que, dos 41 funcionários entrevistados, $61 \%$ não trabalham de carteira assinada e, em se tratando do pagamento por produção, apenas $10 \%$ afirmaram receber algum tipo de remuneração a mais pelo rendimento do trabalho ou pela qualidade sanitária dos animais. Conforme Fischer, Marini e Filippim (2016), a informalidade é um fator que contribui para a insatisfação dos funcionários.

\section{Considerações finais}

Nesta pesquisa, foi possível observar a baixa incidência de práticas de gestão de pessoas na pecuária leiteira da região do Agreste de Pernambuco, sobretudo as baixas gestão e capacitação da mão de obra do setor. Avaliar elementos de gestão, controle, rentabilidade, qualidade e bem-estar dos colaboradores é fundamental para a eficiência e o desenvolvimento das propriedades, pois a eficiência produtiva está intimamente ligada à qualidade da mão de obra da propriedade. Observamos, ainda, ausência de feedback entre gestores e funcionários, ausência de treinamento e muita informalidade, fatores estes que cada vez mais são exigidos pelo mercado e não devem ser negligenciados. 


\title{
People management in the milk proprieties: an analysis in the Agreste of Pernambuco
}

\begin{abstract}
The objective of the article is to evaluate people management practices among the training, remuneration and performance evaluation techniques in the harsh region of the State of Pernambuco. Implementing people management strategies is fundamental to the success of organizations, since the necessary changes in the company will only be effective and legitimate based on the involvement of people. To this end, 41 employees and 30 managers from cities located in the dairy basin of the state of Pernambuco were interviewed. The results pointed out the inexistence of people management techniques in the study region, low training rate, dissatisfaction of employees and high level of informality. The absence of feedback between managers and employees, which resulted in $85 \%$ employee dissatisfaction. However, it is important to emphasize that focusing on people management, stimulating the commitment of the workforce to the properties, generating motivation and training, are key factors for the growth of dairy farming.
\end{abstract}

Keywords: Manpower. Management. Human resources. Dairy farming.

\section{Gestión de personas en propiedades lácteas: un análisis en el Pernambuco Agreste}

\begin{abstract}
Resumen
El objetivo del artículo es evaluar las prácticas de gestión de personas entre las técnicas de capacitación, remuneración y evaluación del desempeño en la dura región del estado de Pernambuco. Implementar estrategias de gestión de personas es fundamental para el éxito de las organizaciones, ya que los cambios necesarios en la empresa solo serán efectivos y legítimos en base a la implicación de las personas. Para ello, se entrevistaron 41 empleados y 30 gerentes de ciudades ubicadas en la cuenca lechera del estado de Pernambuco. Los resultados señalaron la inexistencia de técnicas de gestión de personas en la región de estudio, baja tasa de capacitación, insatisfacción de los empleados y alto nivel de informalidad. La ausencia de retroalimentación entre gerentes y empleados, lo que resultó en un $85 \%$ de insatisfacción de los empleados. Sin embargo, es importante resaltar que enfocarse en la gestión de personas, estimular el compromiso de la fuerza laboral con las propiedades, generar motivación y capacitación, son factores clave para el crecimiento de la ganadería lechera.
\end{abstract}

Palabras clave: Mano de obra. Gestión. Recursos humanos. Ganadería lechera. 


\section{Referências}

ABRANTES, R. S. X. et al. A bovinocultura no sistema agrossilvopastoril. Informativo Técnico do Semiárido, Pombal, PB, v. 10, n. 2, p. 69-75, jul./dez. 2016. Disponível em: https://www.gvaa.com. br/revista/index.php/INTESA/article/download/4573/3935. Acesso em: 19 set. 2019.

ASSOCIAÇÃO BRASILEIRA DAS INDÚSTRIAS DE ALIMENTAÇÃO - ABIA. Números do Setor - Faturamento 2018. São Paulo, SP, 2019. Disponível em: https://www.abia.org.br/vsn/anexos/faturamento2018.pdf. Acesso em: 19 set. 2019.

BRASIL. Decreto-lei no 5.452 , de $1^{\circ}$ de maio de 1943. Aprova a Consolidação das Leis do Trabalho. Disponível em: http://www.planalto.gov.br/ccivil_03/decreto-lei/del5452.htm. Acesso em: 03 dez. 2019.

BRASIL. Lei no 605 , de 5 de janeiro de 1949. Dispõe de Repouso semanal remunerado e o pagamento de salário nos dias feriados civis e religiosos. Disponível em: http://www.planalto.gov.br/ ccivil_03/leis/10605.htm. Acesso em: 03 dez. 2019.

BRASIL. Instrução Normativa $n^{0}$ 77, de 26 de novembro de 2018. Disponível em: http://www. in.gov.br/materia//asset_publisher/kujrw0tzc2mb/content/id/52750141/do1-2018-11-30-instrucao-normativa-n-77-de-26-de-novembro-de-2. Acesso em: 19 out. 2019.

CLEOCY, Jr. Pelo desenvolvimento da Pecuária Leiteira. 2019. Disponível em: http://www.baldebranco.com.br/artigo-pelo-desenvolvimento-da-pecuaria-leiteira/. Acesso em: 20 set. 2019.

CNAE; IBGE. Classificação nacional das atividades econômicas. 2019. Disponível em: http:// www.previdencia.gov.br/dados-abertos/estatisticas-cnae-2-0/. Acesso em: 19 out. 2019.

DA SILVA, A. A. O.; BORDIN, R. D. A.; MACHRY, M.; BUENO, R.; DA SILVA, C. A. Gestão do conhecimento e treinamento do trabalhador na pecuária Leiteira. Revista Eletrônica Thesis, São Paulo, ano XIII, n. 25, p. 103-114, 1․ 2016. Disponível em: http://www.cantareira.br/thesis2/ ed_25/materia6.pdf. Acesso em: 15 ago. 2019.

DE CAMARGO, A. C. Desafios da produção de leite. Embrapa Pecuária Sudeste-Capítulo em livro científico (ALICE), 2018. Disponível em: https://www.alice.cnptia.embrapa.br/bitstream/ doc/1092917/1/DesafiosnaProducaodeLeite.pdf. Acesso em: 02 ago. 2019.

FISCHER, A.; MARINI, D.; FILIPPIM, E. S. Perspectivas de agricultores familiares para a permanência na atividade rural. Revista Espacios, v. 37, n. 7, 2016. Disponível em: https://www. revistaespacios.com/a16v37n07/16370710.html. Acesso em: 21 nov. 2019.

GONÇALVES, M. F.; STEFANO, S. R.; BACCARO, T. A. Sustentabilidade Organizacional e suas relações com a Gestão Estratégica de Pessoas: um estudo de caso em uma cooperativa agroindustrial. Revista de Administração da Unimep, v. 15, n. 3, p. 51-73. 2017. Disponível em: http:// www.raunimep.com.br/ojs/index.php/regen/article/download/1228/733. Acesso em: 25 nov. 2019.

MALANSKI, P. D.; HOSTIOU, N.; INGRAND, S. Evolution pathways of employees' work on dairy farms according to task content, specialization, and autonomy. 2016. Disponível em: https://www. researchgate.net/publication/321828157_Evolution_pathways_of_employees'_work_on_dairy_ farms_according_to_task_content_specialization_and_autonomy. Acesso em: $12 \mathrm{dez} .2019$.

MONTEIRO, A. A. et al. Características da produção leiteira da região do Agreste do estado de Pernambuco, Brasil. Semina: Ciências Agrárias, v. 28, n. 4, p. 665-674, 2007. Disponível em: http://www.uel.br/revistas/uel/index.php/semagrarias/article/download/2901/2459. Acesso em: 12 nov. 2019.

Teoria e Evidência Econômica - a. 26, n. 55, p. 186-200, jul./dez. 2020 
PEREIRA, M. A.; DO VALE, S. M. L. R.; MANCIO, A. B. Gestão de recursos humanos em empresas de bovinos de corte no Triângulo Mineiro. Organizações Rurais e Agroindustriais / Rural and Agro-Industrial Organizations, v. 6, n. 1511-2016-131238, p. 61-74. 2004.

RUSSI, L. S.; COSTA E SILVA, E. V.; ZÚCCARI, C. E. S. N. Importância da capacitação de recursos humanos em programas de inseminação artificial. Revista Brasileira de Reprodução Animal, Belo Horizonte, v. 33, n. 1, p. 20-25, 2009. Disponível em: https://www. bvs-vet.org.br/vetindex/periodicos/revista-brasileira-de-reproducao-animal/33-(2009)-1/ importancia-da-capacitacao-de-recursos-humanos-em-programas-de-insemin/.

SANTOS, L. M. dos. Impacto do transporte a granel na contagem Bacteriana. 2019. Monografia (Bacharelado em Zootecnia) - Universidade Federal Rural de Pernambuco, UFRPE, Garanhuns, 2019. Disponível em: http://repository.ufrpe.br/handle/123456789/1090. Acesso em: 12 dez. 2019.

ULLAH, A.; ZHENG, C. The impact of strategic human resource management practices on Australian dairy farm performance. Work Organization and Human Resource Management, p. 55-72, Springer, Cham, 2014. Disponível em: https://www.researchgate.net/publication/300582307_The_Impact_of_Strategic_Human_Resource_Management_Practices_on_Australian_Dairy_Farm_Performance.

YAMAUCHI, F. A gestão de pessoas no setor do Agronegócio: um estudo sobre produtores de amendoim na região da Alta Paulista. Dissertação (Mestrado) - Unesp, Tupã, São Paulo, 2017. Disponível em: https://repositorio.unesp.br/handle/11449/151079. 